\title{
A Method for Catheter Navigation within Realistic Three-Dimensional Cardiac Anatomy
}

\author{
L Rao, C Ding, DS Khoury
}

\author{
Baylor College of Medicine, Houston, TX, USA
}

\begin{abstract}
We recently combined noncontact mapping and intracardiac echocardiography (ICE) in a single cathetersystem that permitted detailed, single-beat, 3-D electricalanatomical imaging of the heart. In the present study we developed a nonfluoroscopic electrode-catheter localization method and integrated it with noncontact electrical-anatomical imaging. The difference in nonfluoroscopic electrode locations compared to ICE was $6.3 \pm 3.4 \mathrm{~mm}$, while error in reproducibility of the nonfluoroscopic method was $0.7 \pm 1.0 \mathrm{~mm}$. Therefore, accurate and reproducible nonfluoroscopic localization and navigation of standard electrode-catheters within true and detailed 3-D images of cardiac anatomy is feasible. This approach may facilitate diagnosis of arrhythmias and advance their therapy.
\end{abstract}

\section{Introduction}

Radiofrequency catheter ablation is widely used for treating several types of cardiac arrhythmias. In order to guide the ablation catheter to regions of interest inside the heart and to deliver the therapy successfully, visualizing the underlying anatomy and accurate three-dimensional positioning of electrode-catheters at specific locations on the endocardium are necessary.

The disadvantages of routine fluoroscopy during catheterization include radiation effects, prolonged procedure duration, limited three-dimensional localization of the catheter, and poor reproducibility. New systems achieve accurate and reproducible three-dimensional positioning of electrode-catheters by employing specialized position sensing mechanisms in the catheter [1-4]. However, present systems do not provide capabilities for imaging the cardiac anatomy and assumptions must often be made in regards to electrode locations with respect to underlying anatomical structures.

We recently developed a novel cardiac catheter imaging system and tested it in the canine intact beating heart [5]. The system was the first to efficiently integrate noncontact electrical and anatomical imaging by combining a custom multielectrode probe and a standard intracardiac echocardiography (ICE) catheter. This noncontact imaging technique accurately reconstructed single-beat endocardial surface electrograms from measured probe electrograms, provided detailed images of cardiac anatomy based on ICE, and digitally fused both modalities to produce realistic, three-dimensional electrical-anatomical images of the beating heart. The objective of the present study was to develop a nonfluoroscopic electrode-catheter positioning method that could be integrated with noncontact electricalanatomical catheter imaging.

\section{Methods}

\subsection{Experimental model}

A catheter-system, comprised of a 9-F sheath carrying a coaxial noncontact 64-electrode lumen-probe (outer diameter $=7 \mathrm{~mm}$ ) on the outside and a central ICE catheter $(9 \mathrm{~F}, 9 \mathrm{MHz})$ on the inside, was placed inside the heart cavity. Standard, contact electrode-catheters were fixed at various endocardial sites in the LV of 3 dogs and in the RA of 2 other dogs (total = 10 different sites). To reconstruct the endocardial anatomy, the ICE catheter was pulled back inside the sheath in 2-mm steps and twodimensional images were acquired while gating to ECG and respiration. Contact electrode locations and endocardial anatomy were identified by ICE as illustrated in Figure 1. The coaxial noncontact probe was then

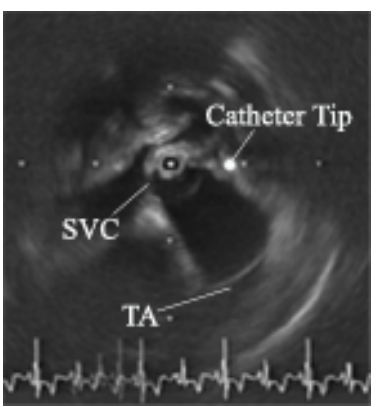

Figure 1. Anatomical imaging in the RA and electrodecatheter localization by ICE. 
advanced into the heart cavity by sliding it over the sheath to fixed markers that aligned the probe with the anatomical images. Electrical current pulses (a homing signal) were emitted from the contact tip-electrodes and the generated potentials were simultaneously sensed by all probe electrodes.

\subsection{Mathematical model}

The probe is comprised of electrodes with coordinates $\left(x_{i}, y_{i}, z_{i}\right)(i=1,2, \ldots, n)$, where $n$ is the number of probe electrodes. Probe electrodes inside the cavity represent field points and their layout is known in advance. The source point where current is injected $(\bar{x}, \bar{y}, \bar{z})$ is the contact electrode at the endocardium. The aim of the localization algorithm is to determine the position of the source point $(\bar{x}, \bar{y}, \bar{z})$.

Let the measured probe potentials be represented by vector $V_{m}=\left[V_{m 1}, V_{m 2}, \ldots, V_{m n}\right]^{T}$, where each $V_{m i}$ $(i=1,2, \ldots, n)$ corresponds to potential on each probe electrode. According to the coordinates of the source point and the field points, $V_{i}$ can be computed using

$$
V_{i}=\frac{I_{s}}{4 \pi \sigma r_{i}} \quad(i=1,2, \ldots, n)
$$

where $I_{s}$ is the injected location current, $\sigma$ is the conductivity of the blood, and $r_{i}$ is the distance between the source point $(\bar{x}, \bar{y}, \bar{z})$ and the ith field point $\left(r_{i}=\sqrt{\left(\bar{x}-x_{i}\right)^{2}+\left(\bar{y}-y_{i}\right)^{2}+\left(\bar{z}-z_{i}\right)^{2}}\right)$.

Let the calculated potentials be represented by $V_{c}=\left[V_{c 1}, V_{c 2}, \ldots, V_{c n}\right]^{T}$, where each $V_{c i}(i=1,2, \ldots, n)$ is a function of source point position $\bar{p}=(\bar{x}, \bar{y}, \bar{z})$. That is, $V_{c}$ can be expressed as

$$
V_{c}=F(\bar{p})
$$

The process of localizing the contact electrodes is an inverse problem. Here, we used the least-squares method by minimizing the following function

$$
\begin{aligned}
R(\bar{p}) & =\frac{1}{2} \sum_{i=1}^{n}\left[F(\bar{p})-V_{m}\right]^{2} \\
& =\frac{1}{2}\left[F(\bar{p})-V_{m}\right]^{T}\left[F(\bar{p})-V_{m}\right]
\end{aligned}
$$

In the above equation, the iterative formula for computing $\bar{p}$ follows

$$
\bar{p}_{k+1}=\bar{p}_{k}+\Delta \bar{p}_{k}
$$

where $\bar{p}_{k+1}$ and $\bar{p}_{k}$ are localization results at the $(k+1)$ th and $k$ th iterative computation respectively, and

$$
\Delta \bar{p}_{k}=-\left[\left[F^{\prime}\left(\bar{p}_{k}\right)\right]^{T}\left[F^{\prime}\left(\bar{p}_{k}\right)\right]+\lambda I\right]^{-1}\left[F^{\prime}\left(\bar{p}_{k}\right)\right]^{T}\left[F\left(\bar{p}_{k}\right)-V_{m}\right]
$$

where $\lambda$ is a regularization parameter determined from the Composite Residual and Smoothing Operator (CRESO) method [6], and $I$ is the identy matrix. From an initial estimate of $\bar{p}$, the iterative process above is executed until satisfying $\max (\Delta V) \leq \varepsilon$, where $\Delta V=F\left(\bar{p}_{k}\right)-V_{m}$ and $\varepsilon$ is a very small positive real number.

\section{Results}

Important anatomical structures were visualized in situ during ICE catheter pullback and without fluoroscopy. In the RA, ICE depicted features such as the appendage, crista terminalis, tricuspid annulus, interatrial septum, fossa ovalis, and coronary sinus ostium. In addition, ICE revealed anatomical structures outside the RA such as other cardiac cavities, pulmonary veins, and the aortic valve. Similarly, in the LV, ICE determined the orientation of the LV walls and depicted the anterior and posterior papillary muscles, the interventricular septum, the right ventricle, and the mitral annulus. Threedimensional anatomical images were successfully constructed based on multiple ICE images throughout the cardiac cycle, as illustrated in Figure 2.

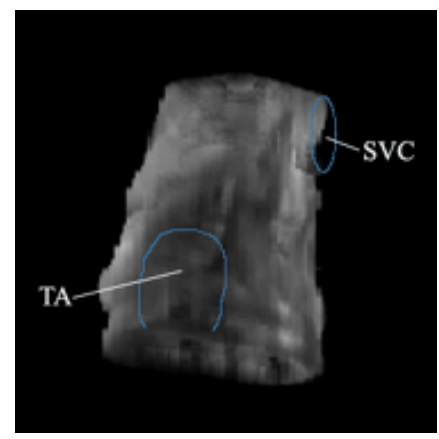

Figure 2. Example of RA geometry by ICE.

\subsection{Comparison of measured and computed catheter locations}

While injecting the location signal through the endocardial catheter tip, the electrical potential inside the cavity was sensed by the probe electrodes. According to our catheter localization method, all 10 catheter tipelectrodes were nonfluoroscopically localized within the three-dimensional geometry, and were correctly matched with corresponding endocardial anatomy. The difference in nonfluoroscopic tip-electrode locations compared to ICE was $6.3 \pm 3.4 \mathrm{~mm}$. One example is shown in Figure 3.

\subsection{Reproducibility of catheter location}

We tested the reproducibility of our system by selecting two separate current signal cycles, and then 


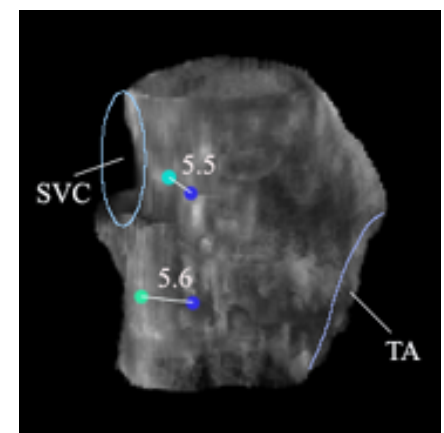

Figure 3. Representative figure depicting the difference between computed and measured locations (in $\mathrm{mm}$ ).

localizing the catheter tips using each cycle information independently. The positioning error between the two cycles was $0.7 \pm 1.0 \mathrm{~mm}$.

\section{Discussion}

We previously developed a catheter system that combined noncontact cavitary mapping with ICE imaging. This system permitted virtual electrical-anatomical imaging of the heart. In the present study, we developed a method for localizing navigational electrode-catheters inside the cavity in concert with the electrical-anatomical imaging catheter. Unlike present mapping systems, our algorithm permitted localizing electrode-catheters within the confines of true images of the endocardial anatomy readily available by ICE. With this approach, details of the heartbeat can be related to underlying anatomy, and ablation therapy can be delivered at specific sites more effectively.

\section{Conclusion}

The present study demonstrated the feasibility of localizing electrode catheters within true and detailed three-dimensional images of cardiac anatomy during catheterization. Integrating this approach with noncontact electrical-anatomical imaging may facilitate diagnosis of arrhythmias and advance their therapy.

\section{Acknowledgement}

This study was supported by grant HL68768 from the National Heart, Lung, and Blood Institute, National Institutes of Health, Bethesda, Maryland.

\section{References}

[1] Gepstein L, Hayam G, Ben-Haim SA. A novel method for nonfluoroscopic catheter-based electroanatomical mapping of the heart. In vitro and in vivo accuracy results. Circulation 1997;95:1611-1622.

[2] Wittkampf FHM, Wever EFD, Derksen R, Wilde AAM, Ramanna H, Hauer RNW, Robles de Medlina EO. New technique for real-time 3-dimensional localization of regular intracardiac electrodes. Circulation 1999;99:13121317.

[3] De Groot NMS, Bootsma M, Van Der Velde ET, Schalij MJ. Three-dimensional catheter positioning during radiofrequency ablation in patients: first application of a real-time position management system. J Cardiovasc Electrophysiol 2000;11:1183-1192.

[4] Schilling RJ, Peters NS, Davies DW. Simultaneous endocardial mapping in the human left ventricle using a noncontact catheter: comparison of contact and reconstructed electrograms during sinus rhythm. Circulation 1998;98:887-898.

[5] Rao L, Khoury DS. System and methods for electricalanatomical imaging of the heart. Proc $2^{\text {nd }}$ Joint EMBS/BMES Conf 2002;1385-1386.

[6] Colli-Franzone P, Guerri L, Taccardi B, Viganotti C. Finite element approximation of regularized solutions of the inverse potential problem of electrocardiography and applications to experimental data. Calcolo 1985;22:91-186.

Address for correspondence:

Dirar S. Khoury, Ph.D.

Baylor College of Medicine

The Methodist Hospital

6535 Fannin St, F551

Houston, TX 77030

dkhoury@bcm.tmc.edu 